[0212-7199 (2008) 25: 1; pp 15-19] ANALES DE MEDICINA INTERNA Copyright (C) 2008 ARAN EDICIONES, S.L.

An. MED INTERnA (Madrid) Vol. 25, N. ${ }^{\circ} 1$, pp. 15-19, 2008

\section{Síndrome de apnea del sueño en la insuficiencia cardiaca. Efecto de la presión positiva continua en la vía aérea}

\author{
J. J. BLANCO PÉREZ, C. ZAMARRÓN SANZ ${ }^{1}$, R. ALMAZÁN ORTEGA², \\ M. GARCÍA GARCÍA ${ }^{3}$, J. LÓPEZ CASTRO, M. TUMBEIRO NOVOA
}

Servicio de Neumología. Complexo Hospitalario de Ourense. ${ }^{I}$ Servicio de Neumología. Complexo Hospitalario Universitario de Santiago. ${ }^{2}$ Unidad de Investigación. Complexo Hospitalario de Ourense. ${ }^{3}$ Servicio de Cardiología. Complexo Hospitalario de Ourense

\section{RESUMEN}

Introducción: Los trastornos respiratorios durante el sueño (TRS) y la insuficiencia cardiaca (IC) son patologías muy comunes que con frecuencia se asocian en el mismo paciente con consecuencias que pueden ser graves.

Objetivo: Conocer la prevalencia de síndrome de apnea del sueño (SAS) en pacientes con IC estable, arritmias asociadas, así como evaluar el efecto del tratamiento con presión positiva continua (CPAP) del SAS sobre la fracción de eyección de ventrículo izquierdo (FE).

Material y métodos: Estudiamos de forma prospectiva a 60 pacientes (50 varones y 10 mujeres) con IC debida a disfunción sistólica (fracción de eyección del ventrículo izquierdo $\mathrm{FE}<45 \%$ ). A todos los pacientes se les realizó un cuestionario específico así como poligrafía respiratoria y un Holter en su domicilio. Se consideró la presencia de SAS cuando el IAH $>15$ y aquellos con IAH $>30$ fueron tratados con CPAP realizando los mismos estudios anteriores, poligrafía y Holter, a los 3 meses.

Resultados: La edad fue de 69,3 $\pm 10,4$ años y el índice de masa corporal (IMC) de $28,5 \pm 4,74 \mathrm{~kg} / \mathrm{m}^{2}$. Un $55 \%$ (33 casos) eran roncadores habituarles y la puntuación en la escala Epworth era de $7,83 \pm 4,8$. La FE fue de $36,3 \pm 7,4 \%$, estando 36 enfermos en clase funcional II, 23 en clase funcional III y un enfermo en clase funcional IV. Un total de 38 pacientes tenían SAS (63\%): 14 (36\%) de tipo central y 24 (64\%) de tipo obstructivo. La FE no mostró diferencias significativas entre el grupo SAS $(36,4 \pm 7,6) v s$. $(35,8 \pm 6,6 \%)$ en el no-SAS. Los pacientes con SAS presentaron más frecuentemente bradicardia sinusal con respecto a los sujetos sin SAS (24\% vs. 9\%). Tras tratamiento con CPAP se observa una mejoría significativa de la FE de 9\%, en los casos de SAS de predominio obstructivo, no observando cambios en los pacientes con SAS de predominio central.

Conclusiones: La prevalencia de síndrome de apnea del sueño en pacientes con insuficiencia cardiaca y disfunción sistólica es muy alta. El tratamiento con CPAP mejora la fracción de eyección de los sujetos con SAS de carácter obstructivo.

PALABRAS CLAVE: Insuficiencia cardiaca. Síndrome de apnea del sueño. Presión positiva continua en la vía aérea.
SLEEP APNEA SYNDROME IN HEART FAILURE. EFFECT OF CONTINUOUS POSITIVE AIRWAY PRESSURE

\section{ABSTRACT}

Introduction: Sleep disordered breathing and heart failure are common disorders that are frequently associated in the same patients which may bring on serious consequences.

Objective: To determine the prevalence of sleep apnea syndrome (SAS) in patients with controlled heart failure and the associated prevalence of arrhythmia. In addition, we to aim to evaluate the effectiveness of continuous positive airway pressure (CPAP) treatment for OSAS on left ventricular ejection fraction $(E F)$.

Material and methods: We prospectively studied 60 patients $(50$ men, 10 women) with heart failure due to systolic dysfunction (left ventricular $E F<45 \%)$. All subjects were classified according the NYHA functional scale and received a specific questionnaire, as well as a home respiratory polygraphy and EKG Holter. A subject was considered to have SAS if their IAH > 15, and, according to standard recommendation, patients with IAH > 30 were treated with CPAP. After 3 months of treatment, the questionnaire, respiratory polygraphy and $E K G$ Holter were repeated.

Results: Age was $69.3 \pm 10.4$ years and the BMI was $28.5 \pm 4.74$ $\mathrm{kg} / \mathrm{m}^{2}$. Of the total sample, $55 \%$ (33 cases) were habitual snorers. The Epworth scale scores were $7.83 \pm 4.8$. The EF was $36.3 \pm 7.4 \%$, and 36 patients were NYHA class II, 23 were class III, and one patient was class IV. A total of 38 patients had SAS (63\%): 14 (36\%) had central sleep apnea and 24 (64\%) had obstructive sleep apnea. EF revealed no significant differences between the SAS and non-SAS groups $(36.42 \pm 7.6 v$ s. $35.81 \pm 6.6 \%$, respectively). The SAS group presented a higher prevalence of bradicardia than the non-SAS group (24\% vs. 9\%). After CPAP treatment, there was a significant improvement in EF (9\%), in cases of obstructive sleep apnea, but no significant improvement was observed in patients with central sleep apnea.

Conclusions: The prevalence of SAS in patients with heart failure due to systolic dysfunction is very high. CPAP treatment improved the EF in patients with obstructive sleep apnea.

KEY WORDS: Heart failure. Sleep apnea syndrome. Continuous positive airway pressure.

Blanco Pérez JJ, Zamarrón Sanz C, Almazán Ortega R, García García M, López Castro J, Tumbeiro Novoa M. Síndrome de apnea del sueño en la insuficiencia cardiaca efecto de la presión positiva continua en la vía aérea. An Med Interna (Madrid) 2007; 25: 15-19.

Trabajo financiado en parte con la beca de ayuda a la investigación (20062007) de la Academia Médico-Quirúrgica y con la colaboración de GASMEDI, S.A. 


\section{INTRODUCCIÓN}

El síndrome de apnea del sueño (SAS) y la insuficiencia cardiaca (IC) son patologías muy comunes, afectando al 2\%-4 y al $1 \%$ respectivamente $(1,2)$. A pesar de los avances en el tratamiento farmacológico, la IC continúa causando repetidas hospitalizaciones, alta morbilidad y mortalidad (3) y alto coste económico (2). Diferentes estudios han mostrado que el SAS se asocia frecuentemente a la IC (4-6) exponiendo al corazón a hipoxia nocturna, incremento de la actividad simpática y de presión arterial así como a fenómenos oxidativos e inflamatorios que pueden facilitar la progresión de esta enfermedad (7). De hecho, estudios epidemiológicos han mostrado que el SAS de carácter obstructivo incrementa el riesgo de desarrollo de IC (8).

El SAS central y/o Cheyne-Stokes es un trastorno muy frecuente en pacientes con fallo cardiaco (5). Sin embargo la influencia étnica, morfología craneofacial, nivel de obesidad o el diferente uso de algunos medicamentos, como los $\beta$ bloqueantes, son factores a tener en cuenta a la hora de generalizar estos resultados no habiéndose realizado ningún estudio de en España que nos aporte información al respecto.

Los pacientes con IC y SAS tienen alta prevalencia de fibrilación auricular y arritmias ventriculares $(9,10)$ así como mayor grado de disfunción ventricular izquierda que los enfermos sin SAS, factores que se han asociado con aumento de la mortalidad (6). Estos pacientes presentan además una menor somnolencia diurna, no observándose relación entre puntuación de la escala de Epworth y la severidad de la apnea (11).

Diferentes estudios han mostrado que en pacientes con IC y SAS obstructivo, el tratamiento con CPAP produce una mejoría de la función sistólica de ventrículo izquierdo $(11,12)$ y de las arritmias $(13,14)$. Por tanto, podría esperarse que dada la alta prevalencia de SAS obstructivo en pacientes con fallo cardiaco, la CPAP podría constituir un tratamiento básico (1318). Sin embargo, esta posibilidad terapéutica no ha sido incluida todavía en las guías de tratamiento del fallo cardiaco (2). De hecho, en el caso de síndrome de apnea central si bien la CPAP puede mejorar la función cardiovascular, recientemente se ha visto que no influye en otros parámetros, entre ellos la supervivencia (18).

En este estudio pretendemos conocer la prevalencia de SAS en pacientes con IC estable, arritmias asociadas, así como evaluar el efecto de la CPAP sobre la fracción de eyección de ventrículo izquierdo.

\section{MATERIAL Y MÉTODOS}

Se incluyeron de forma prospectiva 60 pacientes (50 varones y 10 mujeres) a los que se les realizó un estudio ecocardiográfico entre Enero de 2006 y Diciembre de 2006. Todos ellos presentaban IC debida a disfunción sistólica (fracción de eyección del ventrículo izquierdo $\mathrm{FE}<45 \%$ ) asociado a miocardiopatía dilatada, 38 de etiología isquémica y 22 no isquémica, con estabilidad clínica y sin cambios en el tratamiento en las últimas 8 semanas. Se excluyeron pacientes con angina inestable, enfermedad cardiaca congénita o valvular primaria. Asimismo tampoco se incluyeron pacientes sometidos a tratamiento con teofilinas o enfermedades pulmonares graves.

El protocolo seguido incluía un examen físico completo, registro de medicación, determinación de la disnea según la clase funcional del la NYHA, escala Epworth de somnolencia, así como la realización de poligrafia respiratoria y Holter de arritmias en el domicilio del paciente. Utilizamos un polígrafo respiratorio $\left(\right.$ Embleta $^{\circledR}$ ), recientemente validado (19) y Holter de arritmias (Philips Zymed ${ }^{\circledR}$ ) instalados el mismo día. Se realizó análisis manual de la poligrafía (por dos médicos del Servicio de Neumología, que desconocían la gravedad de la miocardiopatía y en caso de desacuerdo se revisaba el registro conjuntamente) y del Holter por el Servicio de Cardiología.

Se definió apnea como el descenso de la línea del flujo de más del 50\% sobre el nivel basal que durara más de 10 segundos, hipopnea como clara reducción en la amplitud de $>10$ segundos sin cumplir los criterios de apnea asociado a una desaturación $>3 \%$. Apnea central se definió por el cese de flujo y esfuerzo respiratorio. El número de apneas más hipopneas por hora de estudio se denominó índice de apnea-hipopnea (IAH). Se consideró el caso como SAS de tipo central si la suma de apneas centrales y mixtas era superior al 50\% del total de eventos. Para el diagnóstico de respiración tipo Cheyne-Sytokes se requería el típico patrón "waxing-and-waning". Se consideró la presencia de hipersomnolencia a una puntuación en la escala de Epworth mayor de 10. Siguiendo las indicaciones de la SEPAR, a aquellos pacientes que en el estudio de poligrafía respiratoria mostrara IAH $>30$ se les propuso tratamiento con CPAP. Los pacientes que aceptaron el tratamiento, tras un periodo de adaptación con $6 \mathrm{~cm} . \mathrm{H}_{2} \mathrm{O}$ se les tituló la presión de CPAP en laboratorio de sueño. Después de 3 meses de utilización de la CPAP se reevaluó al paciente registrando de nuevo el IMC, escala de Epworth, grado de disnea, cumplimiento de CPAP, ecocardiograma (FE) y monitorización de arritmias con Holter.

Todos los pacientes aceptaron participar en el estudio y firmaron el consentimiento informado.

\section{ANÁLISIS ESTADÍSTICO}

Se diseñó una base de datos con Data Entry 3.0 en la que se recogieron todas las variables de estudio. Se realizó un estudio descriptivo habitual con media, desviación típica o intervalo de confianza al $95 \%$ para las variables cuantitativas y frecuencias absolutas y relativas para las cualitativas. Para la identificación de los factores de riesgo se realizó análisis bivariante con los test estadísticos Ji cuadrado y t de StudentFisher. Cuando las variables no siguieron una distribución normal se aplicaron los test no paramétricos correspondientes. Un valor de $\mathrm{p}<0,05$ se consideró estadísticamente significativo. El análisis se realizó con el programa SPSS 14 (SPSS, Chicago, IL, EE.UU.).

\section{RESULTADOS}

La edad fue de 69,3 \pm 10,4 años y el índice de masa corporal (IMC) de $28,5 \pm 4,7 \mathrm{~kg} / \mathrm{m}^{2}$. Un $55 \%$ (33 casos) eran roncadores habituales y la puntuación en la escala Epworth fué de $7,83 \pm 4,8$. La FE fue de $36,3 \% \pm 7,4 \%$, estando 36 enfermos en clase funcional II, 23 en clase funcional III y un enfermo en clase funcional IV. La FE no mostró diferencias significativas entre el grupo SAS $(36,42 \pm 7,6 v s .35,81 \pm 6,6 \%)$ en el no-SAS. Un total de 38 pacientes tenían SAS (63\%): 14 (36\%) de tipo central y $24(64 \%)$ de tipo obstructivo. Los 
pacientes con apnea, tanto central como obstructiva tenían IMC más elevado que los pacientes sin apnea (Fig. 1). Como puede verse en la tabla I, el $42 \%$ del grupo SAS y $27 \%$ del grupo sin SAS presentaban hipersomnololencia. La saturación mínima de oxígeno fue menor en el grupo SAS $(75,61 v s$. 80,55) y mayor el CT90 (CT90: $11 \pm 4$ en no SAS y $18 \pm 3$ en SAS. Como muestra la tabla II la prevalencia de arritmias auriculares y ventriculares era similar en ambos grupos excep-

\section{TABLA I}

DATOS ANTROPOMÉTRICOS, CLASE NYHA, FRACCIÓN DE EYECCIÓN DE VENTRÍCULO IZQUIERDO Y POLIGRÁFICO EN RELACIÓN CON EL ÍNDICE DE APENA-HIPOPNEA

\begin{tabular}{|c|c|c|c|}
\hline & $I A H<15(n=22)$ & $\mid A H>15(n=38)$ & $p$ \\
\hline Edad (años) & $68,9 \pm 10,4$ & $69,6 \pm 10,6$ & 0,802 \\
\hline Sexo & $17 \mathrm{H}(77 \%)$ & & \\
\hline $5 \mathrm{M}(23 \%)$ & $33 \mathrm{H}(86 \%)$ & & \\
\hline $5 M(14 \%)$ & 0,338 & & \\
\hline IMC $\left(\mathrm{kg} / \mathrm{m}^{2}\right)$ & $26,9 \pm 4,5$ & $29,4 \pm 4,6$ & 0,056 \\
\hline Roncador habitual & $12(54 \%)$ & $21(55 \%)$ & 0,96 \\
\hline Epworth $\geq 10$ & $6(27 \%)$ & $16(42 \%)$ & 0,25 \\
\hline IAH ( Central) & $0(0 \%)$ & $14(36)$ & 0,003 \\
\hline IAH (Obstructiva) & $22(100 \%)$ & $24(64)$ & 0,003 \\
\hline Disnea (NYHA) I y II & $14(63 \%)$ & $22(58 \%)$ & 0,869 \\
\hline Disnea(NYHA) III y IV & $8(36 \%)$ & $16(42 \%)$ & 0,869 \\
\hline Trat $^{\circ}$ con $\beta$ bloqueantes & $17(77,3 \%)$ & $33(86,8 \%)$ & 0,338 \\
\hline СТ90 & $10,9 \pm 4,4$ & $17,8 \pm 3,7$ & 0,245 \\
\hline SAT $\mathrm{O}_{2}$ mínima & $80,5 \pm 6,3$ & $75,6 \pm 8,4$ & 0,022 \\
\hline $\mathrm{FE}$ & $35,8 \pm 6,6$ & $36,4 \pm 7,6$ & 0,760 \\
\hline Respiración C-S & 3 & 17 & 0,029 \\
\hline
\end{tabular}

Los resultados se expresan como media \pm desviación estándar; IAH: número de apneas e hipopneas por hora; CT90: porcentaje de tiempo que la saturación de oxígeno permanece por debajo de 90; FE: fracción de eyección de ventrículo izquierdo; Respiración C-S: Respiración de Cheyne-Stokes.

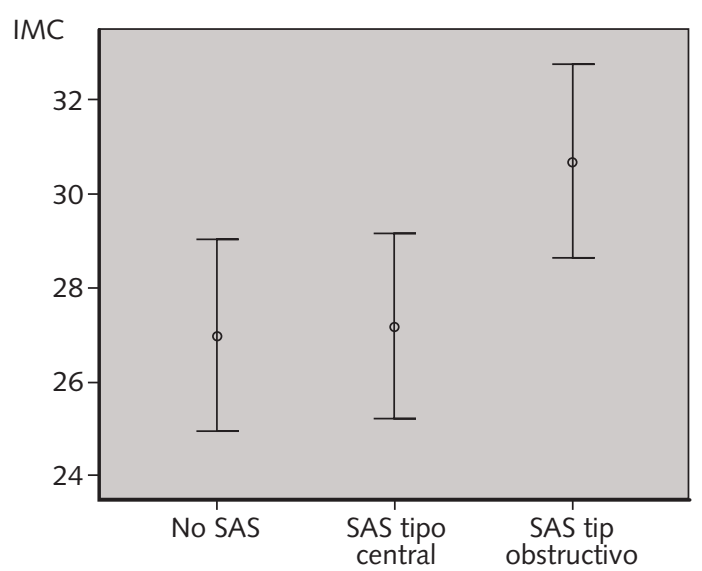

Fig. 1. Diagrama de barras mostrando la relación entre IMC (índice de masa corporal) y SAS (síndrome de apnea del sueño). Se presenta la media e intervalo de confianza al 95\%.
TABLA II

CARACTERÍSTICAS DEL RITMO CARDIACO EN RELACIÓN CON IAH

\begin{tabular}{lccc}
\hline & $I A H<15(n=22)$ & $I A H>15(n=38)$ & $p$ \\
\hline Ritmo sinusal & $16(72 \%)$ & $16(43 \%)$ & 0,028 \\
Bradicardia sinusal & $2(9 \%)$ & $9(23 \%)$ & 0,288 \\
Fibrilación auricular & $2(9 \%)$ & $8(21 \%)$ & 0,401 \\
FC media & $72 \pm 14$ & $69 \pm 17$ & 0,634 \\
Variabilidad FC & $99 \pm 34$ & $97 \pm 33$ & 0,781 \\
Pausas & $5(22 \%)$ & $10(26 \%)$ & 0,757 \\
EV aisladas & $9(41 \%)$ & $10(28 \%)$ & 0,301 \\
EV 100-500 & $8(36 \%)$ & $10(26 \%)$ & 0,413 \\
EV >500 & $4(19 \%)$ & $14(39 \%)$ & 0,120 \\
EV monotópicas & $2(9,5 \%)$ & $3(8 \%)$ & 0,878 \\
EV politópicas & $2(9 \%)$ & $10(30 \%)$ & 0,062 \\
Bigeminismo & $3(14 \%)$ & $2(6 \%)$ & 0,309 \\
Salvas TVNS & $3(13 \%)$ & $3(8 \%)$ & 0,475 \\
Dobletes & $8(36 \%)$ & $14(37 \%)$ & 0,970 \\
Tripletes & $3(13 \%)$ & $8(21 \%)$ & 0,474 \\
Trat ${ }^{\circ}$ con $\beta$ Bbloqueantes & $17(77 \%)$ & $33(86 \%)$ & 0,549 \\
\hline
\end{tabular}

FC: frecuencia cardiaca; EV: extrasístoles ventriculares; TVNS: taquicardia ventricular no sostenida.

\section{TABLA III}

CARACTERÍSTICAS DE FRECUENCIA CARDIACA, DISNEA Y FE TRAS TERAPIA CON CPAP

\begin{tabular}{lccc}
\hline & PRE CPAP & PST CPAP & $p$ valor \\
\hline FC media & 75,30 & 69,50 & $p=0,118$ \\
Variabilidad FC & 87,56 & 129,83 & $p=0,065$ \\
FE & $40,38 \pm 5$ & $44 \pm 10$ & \\
FE (Obstructiva) & $41,43 \pm 3$ & $50,29 \pm 8$ & $p=0,010$ \\
FE (central) & $38,33 \pm 6$ & $36,67 \pm 8$ & $p=0,363$ \\
Disnea (NYHA) & Clase II:10 & Clase I:2 & \\
& Clase III:3 & Clase Il:9 & $p=0,492$ \\
& & Clase III:2 &
\end{tabular}

FE: fracción de eyección del ventrículo izquierdo.

to la bradicardia sinusal y la fibrilación auricular, más frecuentes en el grupo de SAS. De los 22 pacientes a quienes se ofreció el tratamiento con CPAP, fue aceptado en 16 y con adecuado cumplimiento en 13 . La variabilidad cardiaca se incrementó tras CPAP sin que la frecuencia cardiaca se modificase significativamente y la FE pasó de 40,38 \pm 5 a $44 \pm 10$ (Tabla III), aunque la mejoría ocurrió solo en los casos de apnea obstructiva: 41,43 \pm 3 a 50,29 8 (Fig. 2).

\section{DISCUSIÓN}

Nuestro trabajo muestra una alta prevalencia de SAS en pacientes con insuficiencia cardiaca estable no seleccionados por sospecha clínica de SAS. Con mayor frecuencia el SAS fue de tipo obstructivo, con un pequeño grupo con CheyneStokes, mayormente asociado a apnea central. El tratamiento con CPAP mejoró la fracción de eyección de ventrículo izquierdo y la variabilidad de la frecuencia cardiaca. 


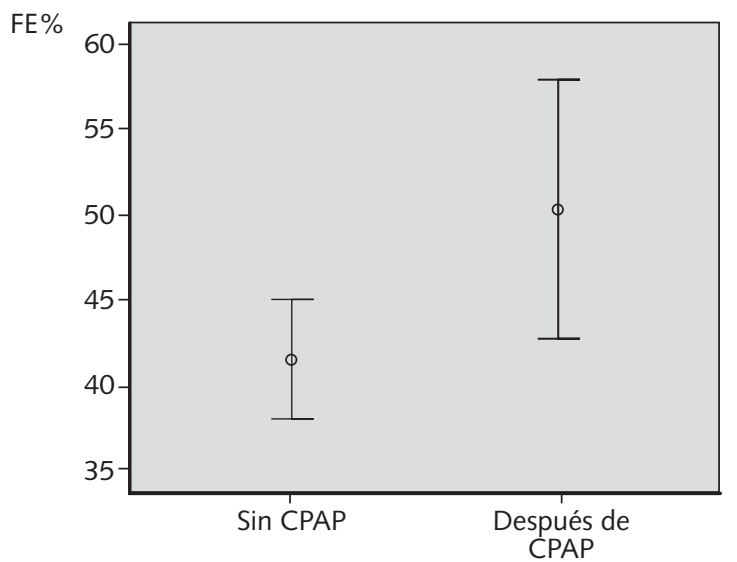

Fig. 2. Diagrama de barras mostrando la fracción de eyección de ventrículo izquierdo antes y después del tratamiento con CPAP en pacientes con SAS obstructivo. Se presenta la media e intervalo de confianza al 95\%.

El predominio de apnea obstructiva en nuestro grupo es similar a lo observado por Sinn y cols., (4) quienes, en un estudio retrospectivo en pacientes que habían sido remitidos por sospecha de trastorno del sueño, encontraron un $61 \%$ de pacientes con IAH > 15 (29 central y $32 \%$ obstructiva). Sin embargo, el estudio prospectivo de Javahery y cols., (5), en pacientes no seleccionados por sospecha de patología de sueño, mostró un $51 \%$ con apnea del sueño con predominio de apnea central (40 central/11\% obstructiva). El hecho de que hayamos empleado cánulas de presión para la detección de apneas-hipopneas que son más sensibles que el térmistor en la detección de alteración respiratoria, pudiera explicar nuestro predominio de apnea obstructiva. Otra explicación puede ser la menor severidad de FE y menor clase NYHA. Además la mayoría de nuestros pacientes seguía tratamiento con $\beta$ bloqueantes lo que puede explicar la menor prevalencia y severidad de SAS central $(20,21)$. A diferencia de los estudios referidos no encontramos diferencias en relación con la FE, la cual era similar en ambos grupos pero sí en relación al IMC, más elevado en pacientes con SAS. Un dato relevante previamente comunicado $(9,22)$, fue que nuestros pacientes con SAS e IC no referían mayor hipersomnolencia con respecto a los que no tenían SAS.

Observamos que la fibrilación auricular, la bradicardia sinusal y la ausencia de ritmo sinusal aparecieron con más frecuencia en el grupo de SAS, sin embargo no se halló más arritmia ventricular a diferencia de lo referido en la literatura (8).

La CPAP tiene efectos importantes efectos hemodinámicas en pacientes con IC, disminuyendo la presión transmural y la actividad simpática. Además, anula los eventos respirato- rios, apneas e hipopneas, y en consecuencia reduce las desaturaciones y los microdespertares. El grupo de pacientes con SAS obstructivo mostró una mejoría de FE del 9\%. Diversos autores demostraron el beneficio del tratamiento con CPAP en SAS de tipo obstructivo $(12,13)$ y central $(17,18)$ en pacientes con IC, al aumentar la FE. Sin embargo hemos de tener en cuenta el efecto deletéreo que puede tener la CPAP en pacientes con apnea central y con baja presión de llenado VI o con fibrilación auricular (23) al reducir el gasto cardiaco. Esta pudo ser la razón de la ausencia de mejoría en la FE de nuestro grupo de apnea central a pesar de haber realizado la titulación adecuada en laboratorio de sueño aplicando una presión de CPAP que oscilaron de 6 a $9 \mathrm{~cm}$ y constatando el control de los eventos respiratorios y de los microdespertares.

La variabilidad de la frecuencia cardiaca está alterada en pacientes con SAS, debido al aumento de actividad simpática. Distintos estudios han mostrado como la disminución de la variabilidad de la frecuencia cardiaca predice morbilidad y mortalidad en pacientes con fallo cardíaco $(24,25)$. En nuestro estudio, el tratamiento con CPAP se asoció a un incremento de la variabilidad de la frecuencia cardiaca, aunque no se modificó el número de arritmias. El aumento de dicha variabilidad si bien no alcanzó rango de significación estadística $(\mathrm{p}=$ $0,065)$ si es clínicamente relevante y puede estar implicado en mejor evolución de su cardiopatía.

Debemos sin embargo reconocer algunas limitaciones. Por una parte es posible que si se hubieran incluido más mujeres la proporción de pacientes con SAS hubiera sido menor. Por otra parte, la reducción del número de pacientes que aceptaron y finalmente cumplieron el tratamiento con CPAP podría explicar la ausencia de mayores diferencias. Consideramos que es necesario incrementar el número de estudios de la patología respiratoria durante el sueño en pacientes con insuficiencia cardiaca, mediante registros domiciliarios independientemente de la presencia de somnolencia diurna excesiva (26) principalmente en presencia de obesidad, bradicardia sinusal y fibrilación auricular. En caso de confirmarse SAS se debería indicar CPAP con adecuada titulación y control clínico y ecocardiográfico de los resultados. Son necesarios estudios randomizados (27) y con mayor número de pacientes para saber si tratando esas patologías se reduce morbilidad y mortalidad de la insuficiencia cardiaca.

En conclusión, en este estudio se ha observado una alta prevalencia de SAS en pacientes con IC estable. Además, el tratamiento con CPAP mejora la fracción de eyección y aumentó la variabilidad de la frecuencia cardiaca en dichos pacientes.

\section{AGRADECIMIENTOS}

A los responsables de la adquisición de las poligrafías respiratorias y holter: D. Carlos Fernández Álvarez y Dña. Josefina García García. 


\section{Bibliografía}

1. Young T, Peppard PE, Gottlieb DI. Epidemiology of obstructive sleep apnea: A population health perspective. Am J Respir Crit Care Med 2002; 165: 1217-39.

2. Thom T, Haase N, Rosamond W, Howard VJ, Rumsfeld J, Manolio T, et al. Heart disease and stroke statistics-2006 update: A report from the American Heart Association Statistics Committee and Stroke Statistics Subcommittee. Circulation 2006; 113: 85-151.

3. Senni M, Tribouilloy CM, Rodehefer RJ, Jacobsen SJ, Evans JM, Bailey KR, et al. Congestive heart failure in the community: Trends in incidence and survival in a 10-year period. Arch Intern Med 1999; 159: 2934.

4. Sin D, Fitzgerald F, Parker JD, Newton G, Floras JS, Bradley TD. Risk factors for central and obstructive sleep apnea in 450 men and woman with congestive heart failure. Am J Respir Crit Care Med 1999; 160: 1101-6.

5. Javaheri S, Parker TJ, Liming JD, Corbett BS, Nishiyama H, Wexer L, et al. Sleep apnea in 81 ambulatory male patients with stable heart failure. Types and their prevalences, consecuences, and presentations. Circulation 1998; 97: 2154-9.

6. Naughton MT, Bradley TD. Sleep apnea in congestive heart failure. Clin Chest Med 1998; 19: 99-113.

7. Bradley TD, Floras JS. Sleep apnea and heart failure. I: Obstructive sleep apnea. Circulation 2003; 107: 1671-8.

8. Shahar E, Whitney CW, Redline S, Lee ET, Newman AB, Javier Nieto $\mathrm{F}$, et al. Sleep-disordered breathing and cardiovascular disease: Crosssectional results of the Sleep Heart Health Study. Am J Respir Crit Care Med 2001; 163: 19-25.

9. Gula LJ, Krahn AD, Skanes AC, Yee R, Klein GJ. Clinical relevance of arrhythmias during sleep: Guidance for clinicians. Heart 2004; 90: $347-$ 52 .

10. Ferrier K, Campbell A, Tee B, Richards M, O'Meeghan T, Weatherall M, et al. Chest 2005; 128: 2116-22.

11. Artz M, Young T, Finn L, Skatrud JB, Ryan MC, Newton GE, et al. Sleepiness and sleep in patients with both systolic heart failure and obstructive sleep apnea. Arch Intern Med 2006; 166: 1716-22.

12. Mansfield DR, Gollogly NC, Kaye DM, Richardson M, Bergin P, Naughton MT. Controlled trial of continuous positive airway pressure in obstructive sleep apnea and heart failure. Am J Respir Crit Care Med 2000; 169: 361-6.

13. Kaneko Y, Floras JS, Usui K, Plante J, Tkacova R, Kubo T, et al. Cardiovascular effects of continuous positive airway presure in patients with heart failure and OSA. N Engl J Med 2003; 348: 1233-41.

14. Ryan CM, Usui K, Floras JS, Bradley TD. Effect of continuous positive airway pressure on ventricular ectopy in heart failure patients with obstructive sleep apnea. Thorax 2005; 60: 781-5.

15. Harbison J, O'Reilly P, MeNieholas WT. Cardiac rhythm disturbances in the obstructive sleep apnea syndrome. Effects of nasal continuous positive airway pressure therapy. Chest 2000; 118: 591-5.

16. Takasaki Y, Orr D, Popkin J Rutherford R, Liu P, Bradley TD. Effets of nasal continuous pressure on sleep apnea in congestive heart failure. Am Rev Respir Dis 1989; 140: 1578-84.

17. Sin DD, Logan AG, Fitzgerald FS, Liu PP, Bradley D. Effects of continuous positive airway pressure on cardiovascular outcomes in heart failure patients with and without Cheyne-Stokes respiration. Circulation 2000; 102: 61-6.

18. Bradley TD, Logan AG, Kimoff RL, Series F, Morrison D, Ferguson K, et al. Continuous positive airway pressure for central sleep apnea and heart failure. N Engl J Med 2005; 353: 2025-33.

19. Dingli K, Coleman EL, Vennelle M, Finch SP, Wraith PK, Mackay TW, et al. Evaluation of a portable device for diagnosing the sleep apnea/hipopnea syndrome. Eur Respir J 2003; 21: 253-9.

20. Köhnlein T, Welte T. Does beta-blocker treatment influence central sleep apnea? Respir Med 2007; 101: 850-3.

21. Arzt M, Bradley TD. Treatment of sleep apnea in heart failure. Am J Respir Crit Care Med 2006; 173: 1300-8.

22. Kaneko Y, Floras JS, Usui K, Plante J, Tkacova R, Kubo T, et al. Cardiovascular effects of continuous positive airway presure in patients with heart failure and OSA. N Engl J Med 2003; 348: 1233-41.

23. De Hoyos A, Liu PP, Benard DC, Bradley TD. Haemodynamic effects of continuous positive airway pressure in humans with normal and impaired left ventricular function. Clin Sci (Lond) 1995; 88:173-8.

24. Sumi K, Chin K, Takahashi K, Nakamura T, Matsumoto H, Niimi, A et al. Effect on nCPAP therapy on heart rate in patents with obstructive sleep apnea-hypopnea. Q J Med 2006; 99: 545-53

25. Ponikowski P, Anker SD, Shua TP, Szelemej R, Piepoli M, Adamopoulos $\mathrm{S}$, et al. Depressed heart rate variability as an independent predictor of death ion chronic congestive heart failure secondary to ischemic or idiopathic dilated cardiomyopathy. Am J Cardiol 1997; 79: 1645-50.

26. Quintana-gallego E., Villa-Gil M, Carmona-Bernal C, Botebol-Benhamou G, Martinez- Martinez A, Sanchez-Armengol A, et al. Home respiratory polygraphy for diagnosis of sleep disordered breathing in heart failure. Eur Respir J 2004; 24: 443-8.

27. Wang H, Parker JD, Newton GE, Floras JS, Mak S, Chiu KL, et al. Influence of obstructive sleep apnea on mortality in patients with heart failure. JACC 2007; 49: 1625-31. 